\title{
Proximity Drawings of Outerplanar Graphs * (extended abstract)
}

\author{
William Lenhart ${ }^{1}$, Giuseppe Liatta ${ }^{2}$ \\ ${ }^{1}$ Department of Computer Science, Williams College, Williamstown, MA 01267. \\ lenhartocs . villiams .edu \\ 2 Department of Computer Science, Brown University, Providence, RI 02912-1910. \\ glocs.brown. edu
}

\begin{abstract}
A proximity drawing of a graph is one in which pairs of adjacent vertices are drawn relatively close together according to some proximity measure while pairs of non-adjacent vertices are drawn relatively far apart. The fundamental question concerning proximity drawability is: Given a graph $G$ and a definition of proximity, is it possible to construct a proximity drawing of $G$ ? We consider this question for outerplanar graphs with respect to an infinite family of proximity drawings called $\beta$-drawings. These drawings include as special cases the well-known Gabriel drawings (when $\beta=1$ ), and relative neighborhood drawings (when $\beta=2$ ). We first show that all biconnected outerplanar graphs are $\beta$-drawable for all values of $\beta$ such that $1 \leq \beta \leq 2$. As a side effect, this result settles in the affirmative a conjecture by Lubiw and Sleumer $[15,17]$, that any biconnected outerplanar graph admits a Gabriel drawing. We then show that there exist biconnected outerplanar graphs that do not admit any convex $\beta$-drawing for $1 \leq \beta \leq 2$. We also provide upper bounds on the maximum number of biconnected components sharing the same cut-vertex in a $\beta$-drawable connected outerplanar graph. This last result is generalized to arbitrary connected planar graphs and is the first non-trivial characterization of connected $\beta$-drawable graphs. Finally, a weaker definition of proximity drawings is applied and we show that all connected outerplanar graphs are drawable under this definition.
\end{abstract}

* Research supported in part by the National Science Foundation under grant CCR9423847, by the U.S. Army Research Office under grant 34990-MA-MUR, and by N.A.T.O.- CNR Advanced Fellowships Programme. 


\section{Introduction and Overview}

Proximity drawings have received increasing attention within the graph drawing community (see, e.g., $[15,9,3,17,2,7,14])$ because of their appealing graphical features: Edges are represented by straight lines, vertices not incident to a certain edge are drawn far apart from that edge, and groups of adjacent vertices tend to cluster together. Also, proximity drawings are of practical interest in several application fields, such as pattern recognition and classification, image processing, geographic variation analysis, GIS, and computational morphology. For a survey on the different application areas of proximity drawings see [12].

A proximity drawing of a graph is has pairs of adjacent vertices relatively close together according to some proximity measure, while pairs of non adjacent vertices are relatively far apart. The choice of proximity measure determines the type of proximity drawing.

One type of proximity drawing is the visibility drawing, in which two vertices $u$ and $v$ are close if and only if they are mutually visible along one of $k$ given directions of visibility. Application areas such as VLSI routing and circuit layout have stimulated considerable research on both 2-dimensional and 3-dimensional visibility drawings in which vertices are segments or simple non overlapping polygons and the directions of visibility are parallel to the axes. (see, e.g., $[1,10]$ ).

A different type of proximity is based upon the concept of a region of influence (also referred to as a proximity region). Given two points $u$ and $v$ in the plane, a proximity region of $u$ and $v$ is a portion of the plane, determined by $u$ and $v$, that contains points relatively close to both $u$ and $v$. A region of influence based proximity drawing of a graph $G$ as a straight-line drawing (vertices of $G$ are mapped to distinct points in the plane, and edges to straight-line segments) such that: (i) for each edge $(u, v)$ of $G$, the proximity region of $u$ and $v$ is empty, i.e. it does not contain any other vertex of $G$; and (ii) for each pair of nonadjacent vertices $u, v$ of $G$, the proximity region of $u$ and $v$ contains at least one other vertex of $G$. For example, in a Gabriel drawing [11], the proximity region of $u$ and $v$ is the closed disk having $u$ and $v$ as antipodal points; in a relative neighborhood drawing [18] the proximity region is the intersection of the two open disks centered at $u$ and at $v$ and with distance $d(u, v)$ as radius. Figure 1 (a) is an example of a Gabriel drawing; the dotted disk is the region of influence of two adjacent vertices. Figure 1 (b) is an example of a relative neighborhood drawing; the dotted lune is the region of influence of two non adjacent vertices. A survey of different types of proximity drawings can be found in [5].

\subsection{The Proximity Drawability Problem}

Although proximity drawings have many uses, the study of their combinatorial properties is still in its early stages. For example, region of influence based proximity drawings are usually adopted in pattern recognition to associate a shape (also called skeleton) to a set of points: pairs of points are connected if and only if their proximity region is empty. Clearly, by changing the proximity region one 


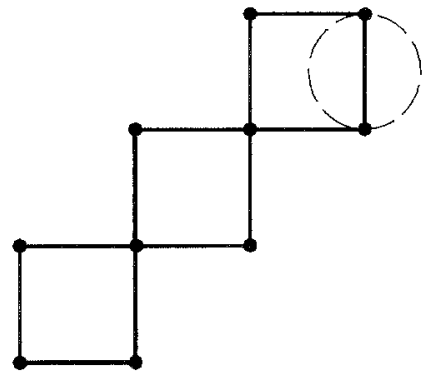

(a)

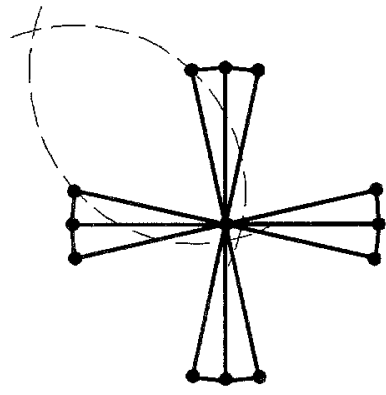

(b)

Fig. 1. (a) A Gabriel drawing, and (b) a relative neighborhood drawing.

can obtain different proximity drawings having the same set of vertices. Surprisingly, for most definitions of proximity the basic properties (such as connectivity, planarity, outerplanarity, convexity etc.) of the proximity drawings that one can possibly obtain on a given point set are not well understood. If, for instance, one whishes to give an outerplanar shape to a point set, he/she needs to know what definition of proximity can give rise to such a shape.

The above discussion motivates the study of the proximity drawability question: given a graph $G$ and a definition of proximity, is it possible to construct a proximity drawing of $G$ ? In this paper we answer the question for biconnected outerplanar graphs with respect to an infinite set of well-known region of influence based proximity drawings called $\beta$-drawings, which were first introduced by Kirkpatrick and Radke [13] in the context of pattern recognition and computational morphology. $\beta$-drawings are based on an infinite family of proximity regions, called $\beta$-regions, each element of the family being identified by a value of the parameter $\beta(0 \leq \beta \leq \infty)$. Given a value of $\beta$, a $\beta$-drawing is a proximity drawing in which the proximity region is the $\beta$-region. These regions are defined precisely in the next section.

For each value of $\beta$, the corresponding $\beta$-region can be either an open or a closed set. A $[\beta]$-drawing is a proximity drawing that adopts a closed $\beta$-region; similarly, a $(\beta)$-drawing adopts an open $\beta$-region. For example, the 1 -region of two points $u$ and $v$ is the disk having $u$ and $v$ as antipodal points; thee 2-region is the intersection of the two disks centered at $u$ and at $v$ and with distance $d(u, v)$ as radius. Hence, a [1]-drawing is a Gabriel drawing and a (2)-drawing is a relative neighborhood drawing (see also Figure 1). In the paper we will use the notation $[\beta]$ - or $(\beta)$-drawing instead of $\beta$-drawing only when the distinction between an open and a closed $\beta$-region is essential for the discussion. We say that a graph is $\beta$-drawable if it has a both open and closed $\beta$-drawings.

The problem of characterizing $\beta$-drawable trees has been studied in $[3,2]$ in the plane, and in [6] in 3-dimensional space. Lubiw and Sleumer $[15,17]$ initiated 
the study of $\beta$-drawability of biconnected outerplanar graphs. They focused on the values $\beta=1$ and $\beta=2$ and showed that every maximal outerplanar graph admits both a (2)-drawing and a [1]-drawing; furthermore, they proved that all biconnected outerplanar graphs are (2)-drawable and conjectured that such graphs are also [1]-drawable.

\subsection{Results}

The main results presented in this paper are listed below.

- We settle in the affirmative the conjecture of Lubiw and Sleumer [15, 17], by showing that all biconnected outerplanar graphs are [1]-drawable. We also extend this result and show that all biconnected outerplanar graphs are $\beta$ drawable for all values of $\beta$ such that $1<\beta<2$. Observe that the interval $1 \leq \beta \leq 2$ is the most interesting for studying the $\beta$-drawability of planar graphs, since, for $\beta>2$, no $\beta$-drawing can have triangular faces; for $\beta<1$, $\beta$-drawings are not guaranteed to be planar [2].

- We study convex $\beta$-drawings. A convex drawing of a planar graph is one in which each face is a convex polygon. Convex drawings are an important object of study in graph drawing because of their aesthetic appeal (see, e.g., $[8,4])$. We provide here a negative result, by showing that there exist biconnected outerplanar graphs that do not admit any convex $\beta$-drawing. We also show that such negative results hold even if the proximity constraint of the drawing is relaxed to the so-called weak $\beta$-proximity model [7]: a weak $\beta$-drawing is such that for every pair of adjacent adjacent vertices the corresponding $\beta$-region is empty, while for pairs of non adjacent vertices the corresponding $\beta$-region may be or may be not empty.

- We extend our investigation to connected graphs. A general theorem is given that provides an upper bound on the number of biconnected components sharing a cut-vertex in a $\beta$-drawable graph, for $\beta \in[0, \infty]$. This is of particular interest since it is the first non-trivial result on the connectivity of $\beta$-drawable graphs. See, e.g., [19] for more on connectivity, As an application of our result, we prove inclusion relations for connected outerplanar $\beta$-drawable graphs with respect to larger classes of graphs.

- We exploit the relationship between $\beta$-drawings, minimum spanning trees, and Delaunay triangulations to show classes of forbidden outerplanar graphs, i.e. outerplanar graphs that are not $\beta$-drawable for $1 \leq \beta \leq 2$. Our approach generalizes to outerplanar graphs previous techniques developed for studying the $\beta$-drawability of trees (see $[3,2]$ ). Also, motivated by the existence of forbidden outerplanar graphs, we consider the representability of outerplanar graphs with the weak $\beta$-proximity model and show that all such graphs admit weak $\beta$-drawings for any given value of $\beta$ in the interval $1 \leq \beta \leq 2$.

Table 1 summarizes the characterization results about the $\beta$-drawability of outerplanar graphs. The entries having a bibliographic reference describe previously known results. All other entries describe results from this paper. 
$\{\mathcal{C O}\},\{B \mathcal{B O}$, and $\{\mathcal{M O}\}$ are the set of all connected outerplanar, biconnected outerplanar, and maximal outerplanar graphs, respectively. $\mathcal{G}_{\mathcal{C O}}(\beta), \mathcal{G}_{\mathcal{B O}}(\beta)$, and $\mathcal{G}_{\mathcal{M O}}(\beta)$ are the classes of connected outerplanar, biconnected outerplanar, and maximal outerplanar $(\beta)$-drawable graphs, respectively. Similarly, $\mathcal{G}_{\mathcal{C O}}[\beta]$, $\mathcal{G}_{\mathcal{B O}}[\beta]$, and $\mathcal{G}_{\mathcal{M O}}[\beta]$ are the classes of connected outerplanar, biconnected outerplanar, and maximal outerplanar $[\beta]$-drawable graphs, respectively. $\mathcal{G}_{k}$ denotes the class of graphs such that the number of biconnected components sharing a cut-vertex is at most $k ; \mathcal{T}_{k}$ is the class of trees whose vertex degree is at most $k$; $\overline{\mathcal{T}}$ is the class of forbidden trees described in [3].

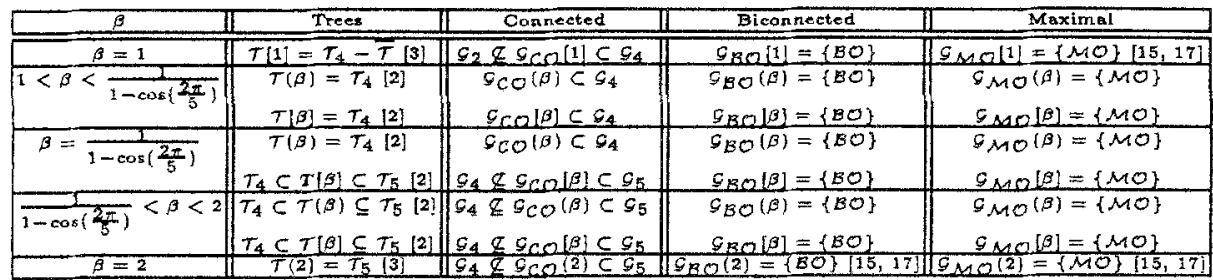

Table 1. Summarizing the characterization results on the $\beta$-drawability of outerplanar graphs for $1 \leq \beta \leq 2$.

\section{Preliminaries}

We review first standard definitions on outerplanar graphs. We then formally define the set of $\beta$-regions. Finally, we introduce the concept of the $\beta$-boundary curve, which will be used for computing $\beta$-drawings of biconnected outerplanar graphs.

\subsection{Outerplanar Graphs}

A planar graph is outerplanar if it has a planar embedding such that all vertices lie on a single face.

For a vertex $u$ in a biconnected outerplanar graph $G$, the fan of $u$, denoted $F_{u}$, is the subgraph induced by all vertices which share a face with $u$. Given an embedding of $G$, let $u_{1}, u_{2}, \ldots, u_{k}$ denote the neighbors of $u$ in clockwise order, and, for each $i<k$, let $u_{i, 1}, u_{i, 2}, \ldots, u_{i, m_{i}}$ denote the vertices of $F_{u}$ non-adjacent to $u$ on the chain from $u_{i}$ to $u_{i+1}$. The edges $u u_{1}$ and $u u_{k}$ are called the first and last edges of $F_{u}$, the edges $u u_{i}$ are called the radial edges, and the remaining edges are called the fan edges. Vertex $u$ is the apex of fan $F_{u}$.

Given a cut-set $S$ of vertices of $G$, let $G_{1}, G_{2}, \ldots, G_{n}$ be the components of $G-S$. The $S$-components of $G$ are the subgraphs of $G$ induced by the sets 
$V\left(G_{i}\right) \cup S$. For a fan edge $e$ of fan $F_{u}$, let $G_{u}(e)$ be the e-component of $G$ not containing $u$.

\subsection{The Set of $\beta$-regions}

Given a pair $x, y$ of points in the plane, the open $\beta$-region of influence of $x$ and $y$, and the closed $\beta$-region of influence of $x$ and $y$, denoted by $R(x, y, \beta)$ and $R[x, y, \beta]$ respectively, are defined as follows [13]:

1. For $0<\beta<1, R(x, y, \beta)$ is the intersection of the two open disks of radius $d(x, y) /(2 \beta)$ passing through both $x$ and $y . R[x, y, \beta]$ is the intersection of the two corresponding closed disks.

2. For $1 \leq \beta<\infty, R(x, y, \beta)$ is the intersection of the two open disks of radius $\beta d(x, y) / 2$, centered at the points $(1-\beta / 2) x+(\beta / 2) y$ and $(\beta / 2) x+(1-\beta / 2) y$. $R[x, y, \beta]$ is the intersection of the two corresponding closed disks.

3. $R(x, y, \infty)$ is the open infinite strip perpendicular to the line segment $\overline{x y}$ and $R[x, y, \infty]$ is the closed infinite strip perpendicular to the line segment $\bar{x} \bar{y}$.

4. $R(x, y, 0)$ is the empty set; $R[x, y, 0]$ is the line segment connecting $x$ and $y$.

Geometric quantities used to analyze $\beta$-drawings are the two angles $\alpha(\beta)$ and $\gamma(\beta)$, defined as follows.

1. $\alpha(\beta)=\inf \{\angle x z y \| z \in R[x, y, \beta]\}$.

2. $\gamma(\beta)$ is only defined for $\beta \geq 2$, and $\gamma(2)=\frac{\pi}{3}$. For $\beta>2$, let $z \neq y$ be a point on the boundary of $R[x, y, \beta]$ such that $d(x, y)=d(x, z)$. Then $\gamma(\beta)=\angle z x y$. short.

In the following property, angles $\alpha(\beta)$ and $\gamma(\beta)$ are denoted as $\alpha$ and $\gamma$, for

\section{Property $2.1[2]$}

The value $\beta$ is related to angles $\alpha$ and $\gamma$ as follows.

$-\beta=\sin \alpha$ for $0 \leq \beta \leq 1$ and $\frac{\pi}{2} \leq \alpha \leq \pi$.

$-\beta=\frac{1}{1-\cos \alpha}$ for $1<\beta \leq 2$ and $0 \leq \alpha<\frac{\pi}{2}$.

$-\beta=\frac{1}{\cos \gamma}$ for $2<\beta \leq \infty$ and $\frac{\pi}{3} \leq \gamma \leq \frac{\pi}{2}$.

\subsection{The $\beta$-Boundary Curve}

Before we can present our algorithm for producing $\beta$-drawings of biconnected outerplanar graphs, we need to introduce and study the following curve.

Definition 1. Given $\beta \in[1,2]$ and points $u$ and $v$ in the plane, the $\beta$-boundary of $u$ with respect to $v$ is the curve $z=\mathcal{C}_{u, v, \beta}(\theta)$ where $z$ is the point in the plane such that $\angle v u z=\theta$ and $v$ is on the boundary of $R[u, z, \beta]$. We assume that $-\pi / 2<\theta<\pi / 2$ and that a positive angle corresponds to $\triangle u v z$ forming a clockwise cycle. 
For example, $\mathcal{C}_{u, v, 1}(\theta)$ is the line through $v$ orthogonal to the segment $\overline{u v}$. In this paper, we will be concerned with only those $\theta$ in the range $[-\pi / 4, \pi / 4]$.

Lemma 2. For $\beta \in[-\pi / 4, \pi / 4]$, if $u=(0,0)$ and $v=(0,1)$, then

$$
\mathcal{C}_{u, v, \beta}(\theta)=\frac{2\left(\sqrt{1-(2 / \beta-1)^{2} \sin ^{2} \theta}-(2 / \beta-1) \cos \theta\right.}{\beta\left(1-(2 / \beta-1)^{2}\right)}(\sin \theta, \cos \theta) .
$$

Sketch of proof: Let $z=\mathcal{C}_{u_{v}, \boldsymbol{v}}(\theta)$ and let $p$ be the center of the circle which defines the portion of the boundary of $R[u, z, \beta]$ containing $v$ and $z$. Since $(\sin \theta, \cos \theta)$ is the unit vector in the direction of $z$ from $u$, we need only determine $|z|$ in order to get a formula for $z$. Let $w=|\overline{u p}|$ and $l=|\overline{p z}|$; then $w+l=|z|$ To determine $w$ and $l$, note that by definition, $w / l=2 / \beta-1$. Using the fact that $|y|=1$ and $|\overline{y p}|=|\overline{p z}|$, it is easy to show that

$$
w+l=\frac{2}{\beta \sqrt{1-(2 / \beta-1)^{2} \sin ^{2} \theta}-(2 / \beta-1) \cos \theta} .
$$

After this, simple algebra suffices to establish the formula of the lemma.

The next lemma describes some important properties of $\mathcal{C}_{u, v, \beta}(\theta)$.

Lemma 3. For $\beta \in(1,2)$, the curve $\mathcal{C}_{u, v, \beta}(\theta)$ is convex in the range $-\pi / 4<$ $\theta<\pi / 4$ and the tangent to $\mathcal{C}_{u, v, \beta}(\theta)$ at $\theta=0$ is orthogonal to $\overline{u v}$.

Sketch of proof: It suffices to show the following.

1. $\mathcal{C}_{u, v, \beta}(\theta)$ has non-zero curvature on $(0, \pi / 4)$ and $(-\pi / 4,0)$,

2. The tangent to $\mathcal{C}_{u, v, \beta}(\theta)$ for all sufficiently small $\theta>0$ is negative, and

3. The tangent to $\mathcal{C}_{u, v, \beta}(\theta)$ for all sufficiently small $\theta<0$ is positive.

The formula for the curvature of a plane curve $(x(\theta), y(\theta))$ is given by

$$
\frac{\left|x^{\prime} y^{\prime \prime}-y^{\prime} x^{\prime \prime}\right|}{\left.\left(\left(x^{\prime}\right)^{2}+\left(y^{\prime}\right)^{2}\right)\right)^{(3 / 2)}}
$$

It can be shown by elementary (but tedious) analytic and algebraic methods that the curvature of $\mathcal{C}_{u, v, \beta}(\theta)$ is non-zero for $\theta \in(-\pi / 4,0) \cup(0, \pi / 4)$, for all $\beta \in(1,2)$. Similar, but simpler, methods can be used to establish properties 2 and 3 .

\section{Proximity Drawings of Biconnected Outerplanar Graphs}

We start by showing how to compute a $\beta$-drawing of a fan. We then show how to compute a $\beta$-drawing of any biconnected outerplanar graph. Finally, we study convexity prperties of $\beta$-drawings of outerplanar graphs. For brevity, we will sometimes use the expression "to $\beta$-draw a graph $G$ " instead of the expression "to compute a $\beta$-drawing of a graph $G$ ". 


\subsection{How to $\beta$-draw a Fan}

We begin with a technical lemma about $\beta$-drawings.

Lemma 4. Given $\beta, 1<\beta<2$, and two graphs $G_{1}$ and $G_{2}$ whose intersection consists of a single edge uv, let $\Gamma_{1}$ and $\Gamma_{2}$ be $\beta$-drawings of graphs $G_{1}$ and $G_{2}$ both of which have $u$ drawn at point $a$ and $v$ at point $b$. If $\Gamma_{1}$ and $\Gamma_{2}$ are on opposite sides of the line determined by ab, and the union of the two drawings lies in a convex region which has both $a$ and $b$ on its boundary, then $\Gamma_{1} \cup \Gamma_{2}$ is a $\beta$-drawing of $G_{1} \cup G_{2}$.

\section{Proof omitted in abstract.}

Lemma 5. Let $F_{u}$ be a fan with apex $u$ such that $u u_{1}$ is the first edge of $F_{u}$ and let $\beta$ be such that $1 \leq \beta<2$. Then $F_{u}$ can be $\beta$-drawn in any triangle $\triangle a b c$ having $\angle a b c>\pi / 2$, and $\angle b a c<\pi / 4$, so that vertices $u$ and $u_{1}$ are drawn at a and $b$, respectively, and that the fan edges form a convex chain with the property that given any three vertices $v_{1}, v_{2}, v_{3}$ on the chain, $\angle v_{1} v_{2} v_{3}>\pi / 2$.

Sketch of proof: The proof is by induction on the number of neighbors of $u$. Assume, for the base case, that $u$ has two neighbors $u_{1}$ and $u_{2}$ connected by a chain $u_{1,1}, \ldots, u_{1, m}$ of non-neighbors of $u$. Let $\theta$ be any angle no greater than $\angle b a c$. We describe a $\beta$-drawing of the fan in $\triangle a b c$ as specified in the statement of the lemma having $\angle u_{1} u u_{2}=\theta$. Moreover, this drawing will have the property that $\angle u_{1, m} u_{2}, u<\pi / 2$.

We first describe how to correctly $\beta$-draw vertex $u_{2}$, and then how to $\beta$-draw the chain of fan vertices from $u_{1}$ to $u_{2}$. Consider the ray from $u$ at a positive angle $\theta$ with the segment $u u_{1}$. We guarantee that $u_{2}$ is not in $R\left[u, u_{1}, \beta\right]$ by placing it exterior to $R\left[u, u_{1}, 2\right]$, the boundary of which is $\mathcal{C}_{u, u_{1}, 2}()$. To ensure that $u_{1}$ is not in the region $R\left[u, u_{2}, \beta\right]$, we position $u_{2}$ interior to the curve $\mathcal{C}_{u, u_{1}, \beta}()$. Note that this is possible since $\mathcal{C}_{u, u_{1}, \beta}()$ intersects the ray beyond the point at which the ray is intersected by $\mathcal{C}_{u, u_{1}, 2}()$.

We show now how to $\beta$-draw the chain of fan vertices from $u_{1}$ to $u_{2}$. We need to ensure that the sequence $u_{1}, u_{1,1}, u_{1,2}, \ldots, u_{1, m}, u_{2}$ is convex, that $\angle u_{1, m} u_{2} u<\pi / 2$, and that each region $R\left[u, u_{1, i}, \beta\right]$ contains some vertex of the sequence. We will position the vertices of the chain on the curve $\mathcal{C} u u_{2} 2$, between the line $L$ determined by $u_{1} c$ and the curve $\mathcal{C} u u_{1} \beta$. It is easy to see that $u_{1}, u_{1,1}, u_{1,2}, \ldots, u_{1, m}, u_{2}$ then form a convex chain in which $\angle u_{1, m} u_{2}, u<\pi / 2$. Since the curve $\mathcal{C} u u_{1} \beta$ intersects each segment $u u_{1, i}, u_{1}$ is in $R\left[u, u_{1, i}, \beta\right]$. Finally, it can be easily checked that $\angle u_{1} u_{1, i} u_{2}>\pi / 2$, for every vertex $u_{1, i}$ on the chain; thus the $\beta$-region of any two non-consecutive vertices on the chain contains some vertex of the chain. This completes the proof of the base case; we now consider the induction step.

Consider a fan $F_{u}$ in which $u$ has $k>2$ neighbors. Let $\theta=\angle b a c /(k-1)$. Draw the part of the fan consisting of $u$ and the chain from $u_{1}$ to $u_{2}$ as in the base case. Now, let $c^{\prime}$ be the intersection of the line determined by $u_{1, m} u_{2}$ and the segment $a c$; let $b^{\prime}=u_{2}$. By induction, the fan $F_{u}-\left\{u, u_{1}, u_{1,1}, \ldots, u_{1, m}\right\}$ 
can be $\beta$-drawn in the (obtuse) triangle $a b^{\prime} c^{\prime}$ as specified in the lemma. Now, by Lemma 4 , the union of the $\beta$-drawings of the two smaller fans is a $\beta$-drawing of $F_{u}$. Observe that, because of the way $\Delta\left(a b^{\prime} c^{\prime}\right)$ is defined, the construction guarantees that $\angle u_{1, m} u_{2} u_{2,1}<\pi$. Figure 2(a) shows fan $F_{u}$ drawn within $\triangle a b c$; Figure 2(b) illustrates details of the drawing strategy described above.

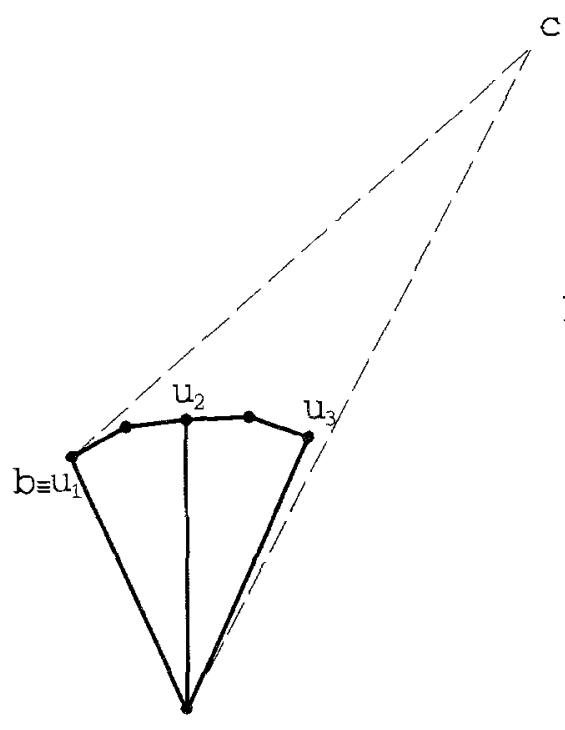

$\mathrm{a}=\mathrm{u}$

(a)

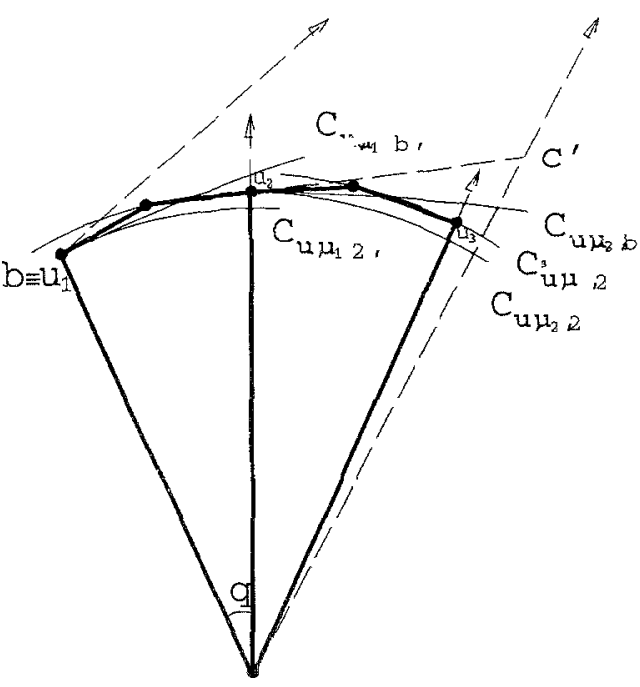

$\mathrm{a}=1$

(b)

Fig. 2. (a) Fan $F_{u} \beta$-drawn inside $\triangle a b c$, and (b) details on how to $\beta$-draw $F_{u}$.

\subsection{How to $\beta$-Draw Biconnected Outerplanar Graphs}

We are now ready to prove the $\beta$-drawability of biconnected outerplanar graphs.

Theorem 6. $\mathcal{G}_{\mathcal{B O}}[1]=\{\mathcal{B O}\}$, and $\mathcal{G}_{\mathcal{B O}}(\beta)=\mathcal{G}_{\mathcal{B O}}[\beta]=\{\mathcal{B O}\}$ for all values of $\beta$ such that $1<\beta<2$.

Sketch of proof: Let $T=\triangle a b c$ be a triangle such that $\angle a b c>\pi / 2$, let $G$ be a biconnected outerplanar graph, and let $u v$ be an edge of $G$. We will show that, for any $\beta \in[1,2), G$ admits a $\beta$-drawing inside $T$ such that $u$ is mapped to $a$ and $v$ is mapped to $b$. Through the proof, we will be using the same notation as in the proof of Lemma 5 . 
By Lemma $5, F_{u}$ can be $\beta$-drawn in triangle $\triangle a b c$ so that vertices $u$ and $v$ are drawn at $a$ and $b$, respectively, and that the fan edges form a convex chain. We will show that, for each fan edge e of $F_{u}$, an obtuse triangle $T_{e}$ having $e$ as a side can be defined such that the following two properties hold,

1. for fan edges $e, e^{\prime}$ such that $e \neq e^{\prime}$ and any points $x \in T_{e}$ and $y \in T_{e^{\prime}}$, some vertex of $F_{u}$ lies in $R[x, y, \beta]$; and

2. if some $T_{e}$ contains a $\beta$-drawing $\Gamma$ of a graph such that $e$ is an edge of $\Gamma$, then no vertex of $\Gamma$ lies in $R\left[u, u_{i}, \beta\right]$, for any $i \leq k$.

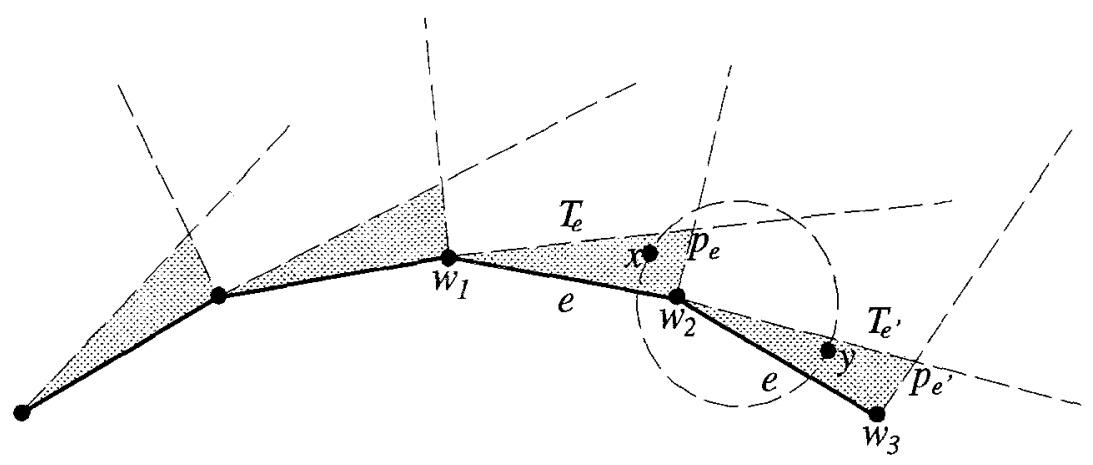

Fig. 3. Illustration for the proof of Theorem 6

This will allow us to $\beta$-draw recursively draw each subgraph $G_{u}(e)$ in $T_{e}$, giving a $\beta$-drawing of $G$ in the original obtuse triangle $T$.

The rest of the proof is concerned with showing that the triangles $T_{e}$ exist. The following two observations can be made about the $\beta$-drawing of $F_{u}$ computed by the algorithm of Lemma 5 ,

- Since the exterior angles along the chain of fan edges of $F_{u}$ are all greater than $\pi$, it is possible to define, for each fan edge $e=w_{1} w_{2}$ a point $p_{e}$ in $\triangle a b c$ such that $w_{1} w_{2} p_{e}$ are vertices of a triangle with $\angle v w p_{e}>\pi / 2$. Further, these points can be positioned so that if $w_{1}, w_{2}, w_{3}$ are consecutive fan vertices, then letting $e=w_{1} w_{2}$ and $e^{\prime}=w_{2} w_{3}, \angle p_{e} w_{2} p_{e^{\prime}}>\pi / 2$ (see Figure 3).

- The drawing algorithm of Lemma 5 does not make any particular assumption about the angle $\angle u_{1} u u_{k}$ between the first edge $u u_{1}$ and the last edge $u u_{k}$ of $F_{u}$, other than $0<\angle u_{1} u u_{k}<\pi / 2$. Also, notice that $\angle u u_{1} u_{1,1}$ can be any obtuse angle less than $\angle a b c$ and that $\angle u_{k-1, m} u_{k} u<p i / 2$. Thus, by using the algorithm of Lemma 5 , it is always possible to $\beta$-draw $F_{u}$ inside $\triangle(a b c)$ so that the following is true: given any vertex $v\left(v \neq u_{1}\right.$ and $\left.v \neq u_{k}\right)$ along the convex chain of fan edges, $\angle u_{1} v u_{k}>\pi / 2$. This implies that given any three vertices $v_{1}, v_{2}, v_{3}$ on the convex chain, $\angle v_{1} v_{2} v_{3}>\pi / 2$. 
The above two observations allow us to prove Property 1 .

To prove Property 2 , let $e=w_{1} w_{2}$ be a fan edge of $F_{u}$, and assume that $T_{e}$ contains a $\beta$-drawing $\Gamma$ of a graph having $e$ as an edge. Since $R\left[u, u_{i}, \beta\right] \cap T_{e}$ $(1 \leq i \leq k)$ is a subset of $R\left[w_{1}, w_{2}, \beta\right], R\left[u, u_{i}, \beta\right]$ cannot contain any vertex of $\Gamma$ (other than possibly $v$ or $w$, if either $v$ or $w$ coincides with $u_{i}$ ).

\subsection{Convexity of Outerplanar $\beta$-drawings}

In this subsection we present a negative result about the convexity of $\beta$-drawings of outerplanar graphs.

We call the outerplanar graph in Figure 4(a) the octagon The vertices of degree four are called corner vertices; the other vertices are side vertices. A [1]-drawing of the octagon is depicted in Figure 4(b).

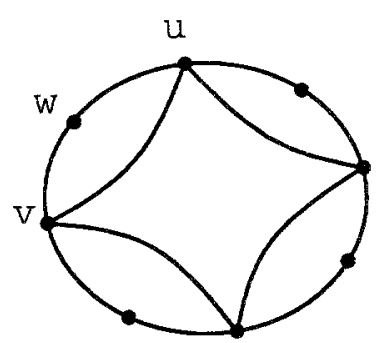

(a)

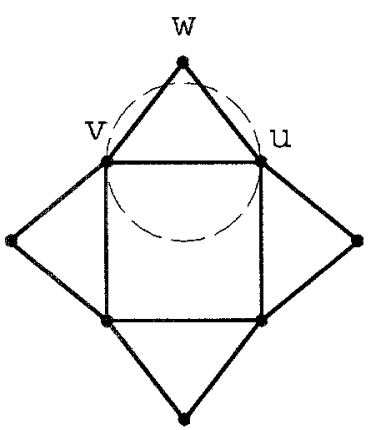

(b)

Fig. 4. (a) An octagon graph $G$, and (b) a [1]-drawing of $G$.

Lemma 7. Any $\beta$-drawing of the octagon for $1<\beta \leq 2$ is such that the external face of the drawing is non-convex. Furthermore, any [1]-drawing of the octagon has non-convex external face.

\section{Sketch of proof:}

Let $G$ be the octagon and let $\Gamma$ be any $\beta$-drawing of $G$ for some value of $\beta$ in the interval stated by the theorem. We show that $\Gamma$ always has two edges incident on a corner vertex that form an angle less than $\pi$ on the external face.

We can make the following two observations on $\Gamma$ (see Figure 4).

1. All vertices of $\Gamma$ must be on the external face. In fact, any 4-cycle in a [1]drawing must be a face (any point inside a quadrilateral $Q$ is contained in at least one of the closed disks having the edges of $Q$ as diameter). Thus a 4-cycle is a face in any $\beta$-drawing with $1<\beta \leq 2$. 
2. For every face $(v, w, u)$ of $\Gamma$ such that $v$ and $u$ are corner and $w$ is side, we have that $\angle v w u<\frac{\pi}{2}$, otherwise $w \in R[v, u, 1]$ (and hence $w \in R[v, u, \beta]$ for larger values of $\beta$ ), contradicting the fact that $v$ and $w$ are adjacent.

Since the outer face of $\Gamma$ is an octagon (Observation 1) and all side vertices are apices of acute angles (Observation 2), it follows that there must be at least one corner vertex of the polygon that is apex of an interior angle larger than $\pi$.

The reasoning of Lemma 7 can be easily extended to weak $\beta$-drawings [7]. A weak $\beta$-drawing is one in which every pair of adjacent vertices has an empty $\beta$ region, while pairs of non-adjacent vertices have $\beta$-regions which might or might not be empty.

Theorem 8. There exists an infinite family of outerplanar graphs which do not admit any convex $\beta$-drawings or convex weak $\beta$-drawings for all values of $\beta$ such that $1<\beta \leq 2$. Furthermore, these graphs do not admit any convex [1]-drawing or convex weak [1]-drawing.

Sketch of proof: The family of graphs consists of all outerplanar graphs having the octagon as a subgraph. The proof of non-convexity of any (weak) $\beta$-drawing of such graphs follows from Lemma 7 .

\section{Proximity Drawings of Connected Outerplanar Graphs}

We first state a general result on the maximum number of biconnected components that can be shared by a cut vertex in any $\beta$-drawable graph and then apply the result to outerplanar $\beta$-drawable graphs.

\subsection{Connectivity of $\beta$-drawable Graphs}

Theorem 9. Given $\beta i n[0, \infty]$, let $(k)$ be the maximum vertex degree in the blockcut-vertex tree of a $(\beta)$-drawable graph. Similarly, let $[k]$ be the maximum vertex degree in the block-cut-vertex tree of a $[\beta]$-drawable graph. The vertex degrees $(k)$ and $[k]$ are related to the value of $\beta$ as follows:

\begin{tabular}{|c|c|c|c|}
\hline & $\beta$ & $(k)$ & {$[k]$} \\
\hline 1 & $0 \leq \beta<2$ & $(k)<\left[\frac{2 \pi}{\alpha(\beta)}\right]$ & {$[k] \leq\left\lfloor\frac{2 \pi}{\alpha(\beta)}\right]$} \\
\hline 2 & $\beta=2$ & $(k)<\left[\frac{2 \pi}{\alpha(\beta)}\right]$ & {$[k]<\left\lfloor\frac{2 \pi}{\gamma(\beta)}\right\rfloor$} \\
\hline 3 & $2<\beta \leq \infty$ & $(k) \leq\left\lfloor\frac{2 \pi}{\gamma(\beta)}\right]$ & {$[k]<\left\lfloor\frac{2 \pi}{\gamma(\beta)}\right\rfloor$} \\
\hline
\end{tabular}


Sketch of proof: The proof generalizes to outerplanar graphs the technique presented in [2] to show upper bounds on the maximum vertex degree of $\beta$ drawable trees. In this extended abstract we show the bounds on the value $(k)$. The technique can be easily extended to show also the bounds on $[k]$. We assume that the graphs in question contain at least one cut-vertex (the theorem is trivially verified otherwise) and start by proving the entries of rows 1 and 2 .

Let $G$ be a $(\beta)$-drawable graph for $0 \leq \beta \leq 2$, and let $\Gamma$ be a $(\beta)$-drawing of $G$. Let $S$ be the set of points in $\Gamma$ that represents the vertices of $G$ and let $v$ be a cut-vertex of $\Gamma$ shared by $(k)$ blocks of $G$. since $0 \leq \beta \leq 2$, every edge of $M S T(S)$ is also an edge of $\Gamma$ (see. e.g. [2]). Consider any two consecutive edges $v x$ and $v y$ of $M S T(S)$ incident with $v$ such that they belong to two distinct blocks of $\Gamma$. We show that the minimum angle between $v x$ and $v y$ is always greater than $\alpha(\beta)$, which implies $(k)<\left\lceil\frac{2 \pi}{\alpha(\beta)}\right\rceil$.

Let $T_{x}$ and $T_{y}$ be the subtrees, containing $x$ and $y$ respectively, obtained by removing $v$ from $M S T(S)$. Let $x^{\prime} \in T_{x}$ and $y^{\prime} \in T_{y}$ be the closest pair of vertices in these two subtrees. Notice that these vertices are not adjacent in $\Gamma$ because they belong to two distinct blocks of $G$. Thus, there must be some vertex $z \in M S T(S)$ such that $z$ is an interior point of $R\left(x^{\prime}, y^{\prime}, \beta\right)$. Clearly $z$ is neither a vertex of $T_{x}$ nor a vertex of $T_{y}$, otherwise, since $0 \leq \beta<2$, we would have that either $d\left(z, x^{\prime}\right)<d\left(x^{\prime}, y^{\prime}\right)$ or $d\left(z, y^{\prime}\right)<d\left(x^{\prime} y^{\prime}\right)$, contradicting the minimality of $d\left(x^{\prime}, y^{\prime}\right)$. (Observe that this condition does not preclude $z$ from being either a vertex of the block of $G$ containing $x^{\prime}$ or of the block of $G$ containing $y^{\prime}$.) Since $z$ is a vertex of $M S T(S)$ and is not adjacent to either $x^{\prime}$ or $y^{\prime}$, we have that $d\left(z, x^{\prime}\right)>d(v, x)$ and $d\left(z, y^{\prime}\right)>d(v, y)$ (if not, $M S T(S)$ would not be a minimum spanning tree). Furthermore, we have that $d\left(x^{\prime}, y^{\prime}\right) \leq d(x, y)$ by the definition of points $x^{\prime}$ and $y^{\prime}$, and that $\angle x^{\prime} z y^{\prime}>\alpha(\beta)$ since $z$ is contained in $R\left(x^{\prime}, y^{\prime}, \beta\right)$. Hence, we can conclude that $\angle x v y>\alpha(\beta)$.

We now prove the correctness of the entry in row 3 . The fact that $(k) \leq\left\lfloor\frac{2 \pi}{\gamma(\beta)}\right\rfloor$ for $2<\beta \leq \infty$ is an immediate consequence of the following claim.

Claim: If a graph is $(\beta)$-drawable for $2<\beta \leq \infty$, then its vertex degree is at most $\left\lfloor\frac{2 \pi}{\gamma(\beta)}\right\rfloor$.

Proof of the claim:. Let $\Gamma$ be any $(\beta)$-drawing for $2<\beta \leq \infty$, let $v$ be a vertex of $\Gamma$ with two consecutive incident edges $v x$ and $v y$. Assume w.l.o.g. that $d(v, x) \leq d(v, y)$. If the angle $\angle x v y<\gamma(\beta)$ then, by the definition of angle $\gamma(\beta)$, we have that $x \in R(v, y, \beta)$, contradicting the assumption that both edges $v x$ and $v y$ belong to $\Gamma$. Thus, the maximum number of edges incident on $v$ cannot be greater than $\left\lfloor\frac{2 \pi}{\gamma(\beta)}\right\rfloor$.

Corollary 10. The following inclusion relations for $\mathcal{G}_{\mathcal{C O}}(\beta)$ and $\mathcal{G}_{\mathrm{CO}}[\beta]$ are true.

1. $\mathcal{G}_{\mathcal{C O}}[1] \subseteq \mathcal{G}_{4}$

2. $\mathcal{G}_{\mathcal{C O}}(\beta) \subseteq \mathcal{G}_{4}$ and $\mathcal{G}_{\mathcal{C O}}[\beta] \subseteq \mathcal{G}_{4}$ for $1<\beta<\frac{1}{1-\cos \left(\frac{2 \pi}{5}\right)}$;

3. $\mathcal{G}_{\mathcal{C O}}(\beta) \subseteq \mathcal{G}_{4}$ and $\mathcal{G}_{\mathcal{C O}}[\beta] \subseteq \mathcal{G}_{5}$ for $\beta=\frac{1}{1-\cos \left(\frac{2 \pi}{5}\right)}$; 
4. $\mathcal{G}_{\mathcal{C O}}(\beta) \subseteq \mathcal{G}_{5}$ and $\mathcal{G}_{\mathcal{C O}}[\beta] \subseteq \mathcal{G}_{5}$ for $\frac{1}{1-\cos \left(\frac{2 \pi}{5}\right)}<\beta<2$

5. $\mathcal{G}_{\mathcal{C O}}(2) \subseteq \mathcal{G}_{5}$.

Figure 5(a), shows a [1]-drawing of an outerplanar graph of class $\mathcal{G}_{4}$. The same drawing is also both a $\beta$-drawing for $1<\beta<\frac{1}{1-\cos \left(\frac{2 \pi}{5}\right)}$ and a $\left(\frac{1}{1-\cos \left(\frac{2 \pi}{5}\right)}\right)$ drawing. Figure $5(\mathrm{~b})$ shows an outerplanar $\left[\frac{1}{1-\cos \left(\frac{2 \pi}{5}\right)}\right]$-drawing of an outerplanar graph of class $\mathcal{G}_{5}$. The same drawing is also both a $\beta$-drawing for $\frac{1}{1-\cos \left(\frac{2 \pi}{5}\right)}<$ $\beta<2$ and a (2)-drawing.

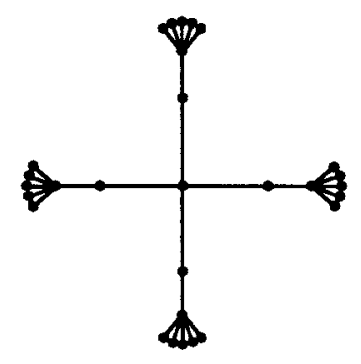

(a)

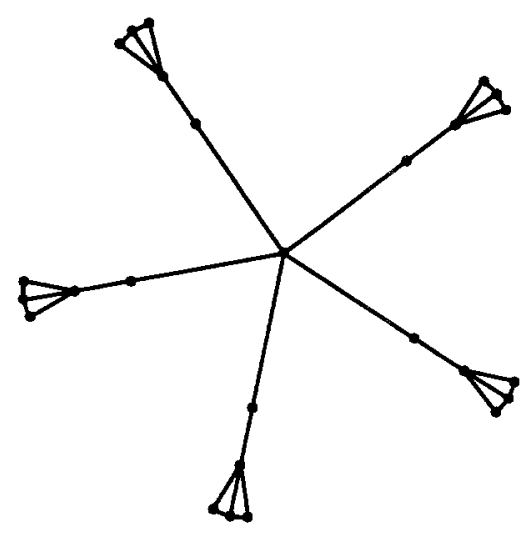

(b)

Fig. 5. (a) A $\beta$-drawing for $1<\beta<\frac{1}{1-\cos \left(\frac{2 \pi}{5}\right)}$, and (b) a $\beta$-drawing for $\frac{1}{1-\cos \left(\frac{2 \pi}{5}\right)}<\beta<2$.

\subsection{Forbidden Outerplanar Graphs and Weak Proximity Drawings}

In [16] it is proven that a [1]-drawing is a subgraph of the Delaunay triangulation of its vertex set. In [3], the relationship between [1]-drawings of trees and Delaunay triangulations is exploited to define classes of forbidden trees (i.e., trees that are not [1]-drawable). We generalize the approach of [3] to show outerplanar graphs that do not admit [1]-drawings even if they satisfy the condition of Theorem 9.

The following lemma relates outerplanar [1]-drawings (i.e. [1]-drawings with all vertices on the external face) to Delaunay triangulations. Its proof is omitted in this extended abstract.

Lemma 11. Let $\Gamma$ be an outerplanar [1]-drawing, let $S$ be the set of vertices of $\Gamma$, and let $D T(S)$ be the Delaunay triangulation of $S$. Let $C$ be a cycle of $D T(S)$ 
that contains at least one vertex of $S$ in its interior. Then there exists an edge $a b \in D T(S)$ and a vertex $p \in S$ such that $a b \notin \Gamma, \triangle(a p b)$ is a face of $D T(S)$, $\triangle(a p b)$ is inside $C$, and $\angle a p b \geq \frac{\pi}{2}$.

Based on the above result, the following lemma can be proved.

Lemma 12. All exterior angles in an outerplanar [1]-drawing of an outerplanar graph are at least $\frac{\pi}{2}$.

An extended octagon graph is a graph constructed by adding $k$ biconnected components to each reflex vertex of the octagon (call this octagon the root of the extended octagon graph). The number $k$ is the degree of the extended octagon graph. For example, the graph of Figure 6(a) is an extended octagon graph of degree 1, the graph of Figure 6(b) is an extended octagon graph of degree 3.

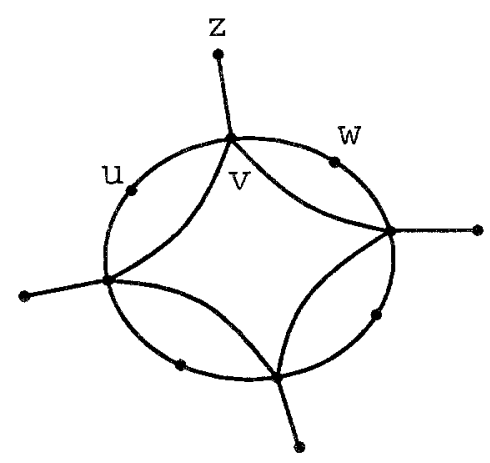

(a)

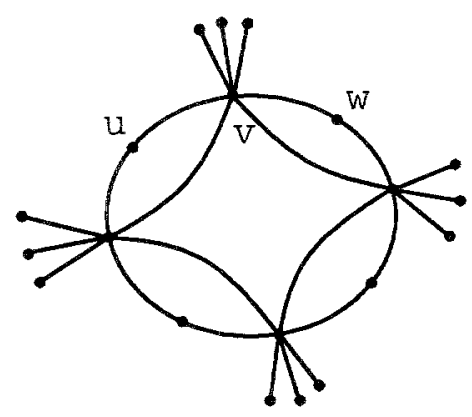

(b)

Fig. 6. (a) An outerplanar graph that does not admit a [1]-drawing, and (b) an outerplanar graph that does not admit a $\beta$-drawing for $1<\beta \leq 2$.

Lemma 13. An extended octagon graph of degree 1 does not admit a [1]-drawing.

Sketch of proof: Let $G$ be an extended octagon graph of degree 1 and let $G^{\prime}$ be its root (see Figure 6 (a)). Suppose $G$ admitted a [1]-drawing $\Gamma$. Observe that, by the same reasoning as in the proof of Lemma $7, \Gamma$ is an outerplanar [1]-drawing. Also, as in Lemma 7, there exist two consecutive exterior edges $v u$, $v w$ of $G^{\prime}$ such that $v$ is a corner vertex and $\angle u v w<\pi$. Let $v z$ be an edge of $G$ not in $G^{\prime}$; we have that either $\angle u v z<\frac{\pi}{2}$ or $\angle z v w<\frac{\pi}{2}$ (or both), contradicting Lemma 12.

Lemma 14. An extended octagon graph of degree 3 does not admit a $\beta$-drawing for any value of $\beta$ such that $1<\beta \leq 2$. 
Sketch of proof: Let $G$ be an extended octagon graph of degree 3 and let $G^{\prime}$ be its root (see Figure 6 (b)). Suppose $G$ admitted a $\beta$-drawing $\Gamma$ for some $\beta \in[1,2]$. With the same reasoning as in the proof of the previous lemma, we have that there exist two consecutive exterior edges $v u, v w$ of $G^{\prime}$ such that $v$ is a reflex vertex and $\angle x v y<\pi$. By the technique in the proof of Theorem 9 , it is an easy task to show that the number of blocks that share $v$ (other than $G^{\prime}$ ) and that can be drawn between edges $u v$ and $v w$ in $\Gamma$ is at most $\left\lfloor\frac{\pi}{\alpha(\beta)}\right\rfloor<3$, thus contradicting the assumption that $\Gamma$ is a $\beta$-drawing of $G$.

We are now in a position to prove the main result of this section. The proof of the following theorem directly follows from Lemmas 13, 14, and from the observation that the maximum vertex degree of the block-cut-vertex tree of an extended octagon graph of degree $k$ is $k+1$.

Theorem 15. For $\beta \in[1,2]$, the sets $\mathcal{G}_{\mathcal{C O}}(\beta)$ and $\mathcal{G}_{\mathcal{C O}}[\beta]$ vary as shown in Table 1 .

Motivated by the existence of forbidden connected outerplanar graphs, we consider the the representability of outerplanar graphs with weak $\beta$-drawings.

Theorem 16. Every connected outerplanar graph $G$ admits a weak [1]-drawing, and a weak (2)-drawing. Furthermore, $G$ admits a weak $\beta$-drawing for any given $\beta$ such that $1<\beta<2$.

Sketch of proof: There are several possible ways for constructing a weak $\beta$ drawing of a connected outerplanar graph for $1 \leq \beta<2$. For example, one can add edges to the input graph $G$ to make it biconnected, construct a $\beta$-drawing of $G$ by the technique of Theorem 6 and then delete the dummy edges introduced during the make-biconnected step.

\section{Open Problems}

Several questions remain open. For example,

1. Completely characterize the class of $\beta$-drawable connected outerplanar graphs for $1 \leq \beta \leq 2$.

2. The drawing algorithms presented in this paper are based on the real RAM model of computation. It would be interesting to devise numerically robust algorithms for computing proximity drawings of graphs. For example, Theorem 16 shows that weak $\beta$-drawings of outerplanar graphs are realizable. However, it would be interesting to study weak $\beta$-drawings of graphs where the coordinates of the vertices are integer numbers or rational numbers of limited size.

3. Study other aesthetic criteria of $\beta$-drawings, other than convexity. Among them, we find particularly important the area requirement, the aspect ratio, and the ratio of the longest to the shortest edge.

4. Extend the $\beta$-drawability question to the study the of non outerplanar graphs. Theorem 9 could be an useful starting point. 


\section{References}

1. H. Alt, M. Godau, and S. H. Whitesides. Universal 3-dimensional visibility representations for graphs. In Graph Drawing (Proc. GD '95), volume 1027 of Lecture Notes in Computer Science, pages 8-19. Springer-Verlag, 1996.

2. P. Bose, G. Di Battista, W. Lenhart, and G. Liotta. Proximity constraints and representable trees. In Graph Drawing (Proc. GD '95), volume 1027 of Lecture Notes in Computer Science. Springer-Verlag, 1995.

3. P. Bose, W. Lenhart, and G. Liotta. Characterizing proximity trees. Technical Report TR-SOCS 93.9, School of Computer Science, McGill University, 1993.

4. M. Chroback, M. T. Goodrich, and R. Tamassia. Convex drawings of graphs in two and three dimensions. In Proc. 12th Annu. ACM Sympos. on Computational Geometry, pages 319-328, 1996.

5. G. Di Battista, W. Lenhart, and G. Liotta. Proximity drawability: A survey. In Graph Drawing (Proc. GD '95), volume 1027 of Lecture Notes in Computer Science. Springer-Verlag, 1995.

6. G. Di Battista and G. Liotta. Computing proximity drawings of trees in 3-d space. In WADS (Proc. WADS '95). Springer-Verlag, 1995.

7. G. Di Battista, G. Liotta, and S. H. Whitesides. The strength of weak proximity. In Graph Drawing (Proc. GD '95), volume 1027 of Lecture Notes in Computer Science, pages 178-189. Springer-Verlag, 1996.

8. P. Eades and P. Garvau. Drawing stressed planar graphs in three dimensions. In Graph Drawing (Proc. GD '95), volume 1027 of Lecture Notes in Computer Science, pages 212-223. Springer-Verlag, 1996.

9. P. Eades and S. H. Whitesides. The realization problem for euclidean minimum spanning trees is np-hard. In Proc. ACM Symp. on Comp. Geom., 1994.

10. S. Fekete, M. Houle, and S. H. Whitesides. New results on a visibility representation of graphs in 3d. In Graph Drawing (Proc. GD '95), volume 1027 of Lecture Notes in Computer Science, pages 234-241. Springer-Verlag, 1996.

11. K. R. Gabriel and R. R. Sokal. A new statistical approach to geographical analysis. Systematic Zoology, 18:54-64, 1969.

12. J. W. Jaromczyk and G. T. Toussaint. Relative neighborhood graphs and their relatives. In Proceedings of the IEEE, 80, pages 1502-1517, 1992.

13. D. G. Kirkpatrick and J. D. Radke. A fraraework for computational morphology. In G. T. Toussaint, editor, Computational Geometry, pages 217-248. Elsevier, Amsterdam, 1985.

14. W. Lenhart and G. Liotta. Drawing outerplanar minimum weight triangulations. Inform. Process. Lett, 6(12):253-260, 1996.

15. A. Lubiw and N. Sleumer. All maximal outerplanar graphs are relative neighborhood graphs. In Proc. Fifth CCCG, pages 198-203, 1993.

16. D. W. Matula and R. R. Sokal. Properties of gabriel graphs relevant to geographic variation research and the clustering of points in the plane. Geographical Analysis, $12(3): 205-222,1980$.

17. N. Sleumer. Oterplanar graphs as proximity graphs. Master's thesis, University of Waterloo, Watreloo, Canada, 1993.

18. G. Toussaint. The relative neighborhood graph of a finite planar set. Pattern Recognition, 12:229-260, 1980.

19. W. T. Tutte. Connectivity in Graphs. Oxford University Press, 1972. 